\title{
Muséologies
}

Les cahiers d'études supérieures

muséologies

\section{Guerres culturelles et musées d'art aux États-Unis : le cas de Hide/Seek: Difference and Desire in American Portraiture}

\section{Julia Roberge Van Der Donckt}

Volume 6, numéro 1, 2012

URI : https://id.erudit.org/iderudit/1011531ar

DOI : https://doi.org/10.7202/1011531ar

Aller au sommaire du numéro

Éditeur(s)

Association Québécoise de Promotion des Recherches Étudiantes en Muséologie (AQPREM)

\section{ISSN}

1718-5181 (imprimé)

1929-7815 (numérique)

Découvrir la revue

Citer cet article

Roberge Van Der Donckt, J. (2012). Guerres culturelles et musées d'art aux États-Unis : le cas de Hide/Seek: Difference and Desire in American Portraiture. Muséologies, 6(1), 35-53. https://doi.org/10.7202/1011531ar

\section{Résumé de l'article}

Ne faisant pas exception à la résurgence épisodique de conflits idéologiques émanant des guerres culturelles américaines, les institutions artistiques constituent des arènes de prédilection pour l'émergence de certaines polémiques. Dans son article, Julia Roberge Van Der Donckt transpose la théorie sociologique de l'acteur-réseau aux disciplines de la muséologie et de l'histoire de l'art pour faire l'étude de la controverse qui entoura le retrait de l'oeuvre A Fire in My Belly de l'artiste américain David Wojnarowicz alors qu'elle était présentée à la National Portrait Gallery de Washington (DC), dans le cadre de l'exposition Hide/Seek: Difference and Desire in American Portraiture. À l'aide de cet exemple, l'auteure démontre comment la multiplication des interactions entre certains acteurs peut constituer une chaîne de médiations qui influence et façonne une situation tout en menant parfois à l'émergence, l'escalade, puis l'apaisement d'une polémique. L'analyse de cet épisode rend également compte des conséquences fondamentales que génère la recrudescence des guerres culturelles américaines sur la sphère muséale, dont la vulnérabilité du financement public des musées, la censure, la création réactionnaire de modes de diffusions artistiques alternatifs et la remise en question de l'indépendance des musées quant à la présentation et à l'authenticité de leurs contenus d'exposition.
Tous droits réservés (C) Association Québécoise de Promotion des Recherches Étudiantes en Muséologie (AQPREM), 2012
Ce document est protégé par la loi sur le droit d'auteur. L'utilisation des services d'Érudit (y compris la reproduction) est assujettie à sa politique d'utilisation que vous pouvez consulter en ligne.

https://apropos.erudit.org/fr/usagers/politique-dutilisation/ 
Article deux

Guerres culturelles et musées d'art aux États-Unis: le cas de Hide/Seek: Difference and Desire in American Portraiture

Julia Roberge Van Der Donckt 
Ne faisant pas exception à la résurgence épisodique de conflits idéologiques émanant des guerres culturelles américaines, les institutions artistiques constituent des arènes de prédilection pour l'émergence de certaines polémiques. Dans son article, Julia Roberge Van Der Donckt transpose la théorie sociologique de l'acteur-réseau aux disciplines de la muséologie et de l'histoire de l'art pour faire l'étude de la controverse qui entoura le retrait de l'œuvre A Fire in My Belly de l'artiste américain David Wojnarowicz alors qu'elle était présentée à la National Portrait Gallery de Washington (DC), dans le cadre de l'exposition Hide/Seek: Difference and Desire in American Portraiture. À l'aide de cet exemple, l'auteure démontre comment la multiplication des interactions entre certains acteurs peut constituer une chaîne de médiations qui influence et façonne une situation tout en menant parfois à l'émergence, l'escalade, puis l'apaisement d'une polémique. L'analyse de cet épisode rend également compte des conséquences fondamentales que génère la recrudescence des guerres culturelles américaines sur la sphère muséale, dont la vulnérabilité du financement public des musées, la censure, la création réactionnaire de modes de diffusions artistiques alternatifs et la remise en question de l'indépendance des musées quant à la présentation et à l'authenticité de leurs contenus d'exposition.

\footnotetext{
Julia Roberge Van Der Donckt est titulaire d'un baccalauréat en histoire de l'art de l'Université Laval. Après un stage en conservation au Musée des Augustins de Toulouse, elle a obtenu une maîtrise en muséologie de l'Université de Montréal. Cet article est un extrait de son travail dirigé. À l'automne 2011, elle a entamé un doctorat en histoire de l'art à l'Université de Montréal, sous la direction de Suzanne Paquet. Ses recherches portent sur les controverses touchant les expositions d'art contemporain aux États-Unis. julia.rvdd@gmail.com
} 
L'épiphénomène que constituent les guerres culturelles américaines a fait l'objet de nombreuses études au cours des vingt dernières années. Les spécialistes ne sont cependant toujours pas arrivés à un consensus en ce qui concerne l'ampleur exacte des affrontements idéologiques dont il est question ${ }^{1}$. Bien que les positions relatives à ces luttes divergent sensiblement, il est possible de dégager certains aspects récurrents. De manière générale, le vocable culture wars est employé pour désigner la polarisation fondée sur un conflit de valeurs présente dans la société étasunienne depuis la fin de la guerre froide, apparente notamment sur la scène politique, mais également dans de nombreux autres secteurs. Le sociologue James Davison Hunter, considéré comme le responsable de l'entrée de la discussion sur les culture wars dans le cercle scientifique, décrit cette notion de la façon suivante: «I define cultural conflict [...] as political and social hostility rooted in different systems of moral understanding. The end to which these hostilities tend is the domination of one cultural and moral ethos over all others ${ }^{2}$. " Souvent désignées au pluriel, ces guerres se jouent sur une multiplicité de terrains, les institutions artistiques n'y faisant pas exception.

Les aléas de ces dites culture wars ont eu de fortes répercussions sur la sphère muséale américaine au cours des vingt dernières années. Certains établissements sont ainsi devenus la cible de débats houleux, étant le théâtre de luttes opposant progressistes et conservateurs, ce qui mena notamment à une diminution substantielle du financement public des arts et même jusqu'à des poursuites judiciaires ${ }^{3}$. Cet article met en lumière le développement de la controverse toute récente entourant l'exposition Hide/Seek: Difference

1 Voir FIORINA, Morris P. Culture War? The Myth of a Polarized America. New York: Pearson Longman, 2005; et WILLIAMS, Rhys. Cultural Wars in American Politics: Critical Reviews of a Popular Myth. New York: Aldine de Gruyter, 1997.

2 HUNTER, James Davison. Culture Wars: The Struggle to Define America. Making Sense of the Battles over the Family, Art, Education, Law and Politics. New York: Basic Books, 1991, p. 42.

3 Voir WALLIS, Brian, Marianne WEEMS et Philip YENAWINE (dir.). Art Matters: How the Culture Wars and Desire in American Portraiture, présentée à la National Portrait Gallery (NPG) de Washington (DC) du 30 octobre 2010 au 13 février 2011, en relevant les mécanismes qui ont régi la mise en place de cette polémique. Confirmant de manière probante le rôle déterminant exercé par les institutions muséales au sein des guerres culturelles affectant la société américaine, ce cas concentre également des enjeux fondamentaux liés à la censure et aux modes de financement des musées.

À la suite de critiques acerbes émises par des acteurs conservateurs influents, l'œuvre A Fire in My Belly de l'artiste américain David Wojnarowicz fut précipitamment retirée de l'exposition par la direction du groupe Smithsonian, institution publique qui regroupe 19 musées aux États-Unis, dont la National Portrait Gallery. Dès lors, un vaste débat s'ouvrit. Plusieurs organisations artistiques, auteurs et activistes contestèrent ce qu'ils considéraient être un acte de censure injustifié, alors que les détracteurs de l'exposition appelaient à davantage de fermeté en ce qui concerne la gestion financière de la NPG. L'œuvre ne fut finalement pas réintégrée dans Hide/Seek, en dépit de l'insistance de la communauté artistique. Néanmoins, les échanges au sujet de cette exposition ambitieuse aux conséquences inattendues se poursuivirent plusieurs mois après sa fermeture, inaugurant entre autres de nouveaux modes de diffusion et d'échange par l'entremise d'Internet. Après une décennie de relative quiétude, Hide/Seek annoncerait ainsi un retour en force des guerres culturelles sur la scène muséale ${ }^{4}$.

Changed America. New York: New York University Press, 1999; et DUBIN, Steven C. Arresting Images: Impolitic Art and Uncivil Actions. Londres: Routledge, 1991.

4 La dernière controverse majeure à avoir touché les musées d'art américains entourait l'exposition Sensation: Young British Artists from the Saatchi Collection, qui avait été présentée au Brooklyn Museum en 1999. À ce sujet, voir ROTHFIELD, Lawrence (dir.). Unsettling “Sensation.” ArtsPolicy Lessons from the Brooklyn Museum of Art Controversy. New Brunswick: Rutgers University Press, 2001. 


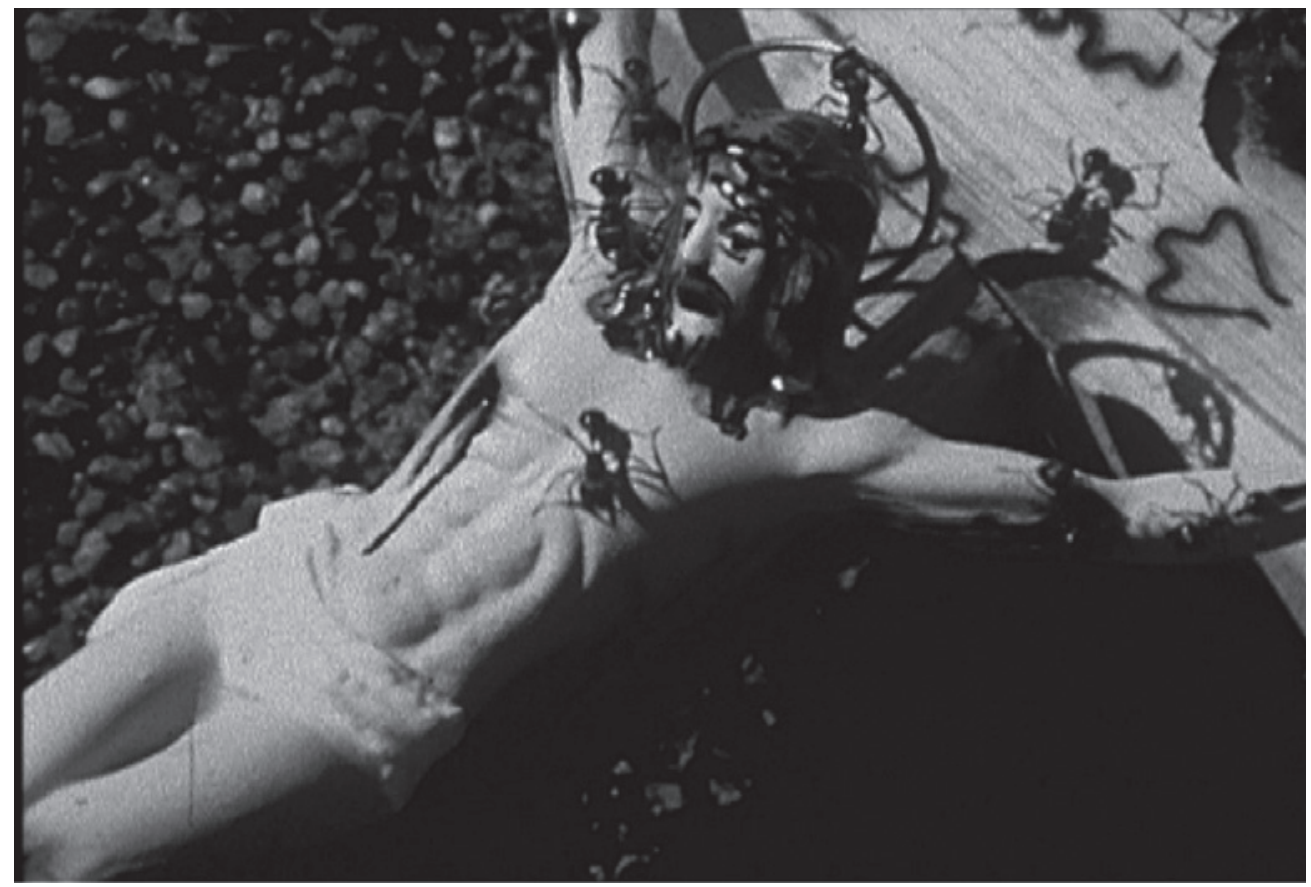

\section{Illustration 1}

Extrait de A Fire in My Belly (Film in Progress),

David Wojnarowicz, 1986-1987, film muet, super $8 \mathrm{~mm}$, noir et blanc et couleur, transféré sur vidéo.

Reproduit avec la permission de The Estate of David Wojnarowicz et PPOW Gallery, New York et The Fales Library and Special Collections, New York University. 


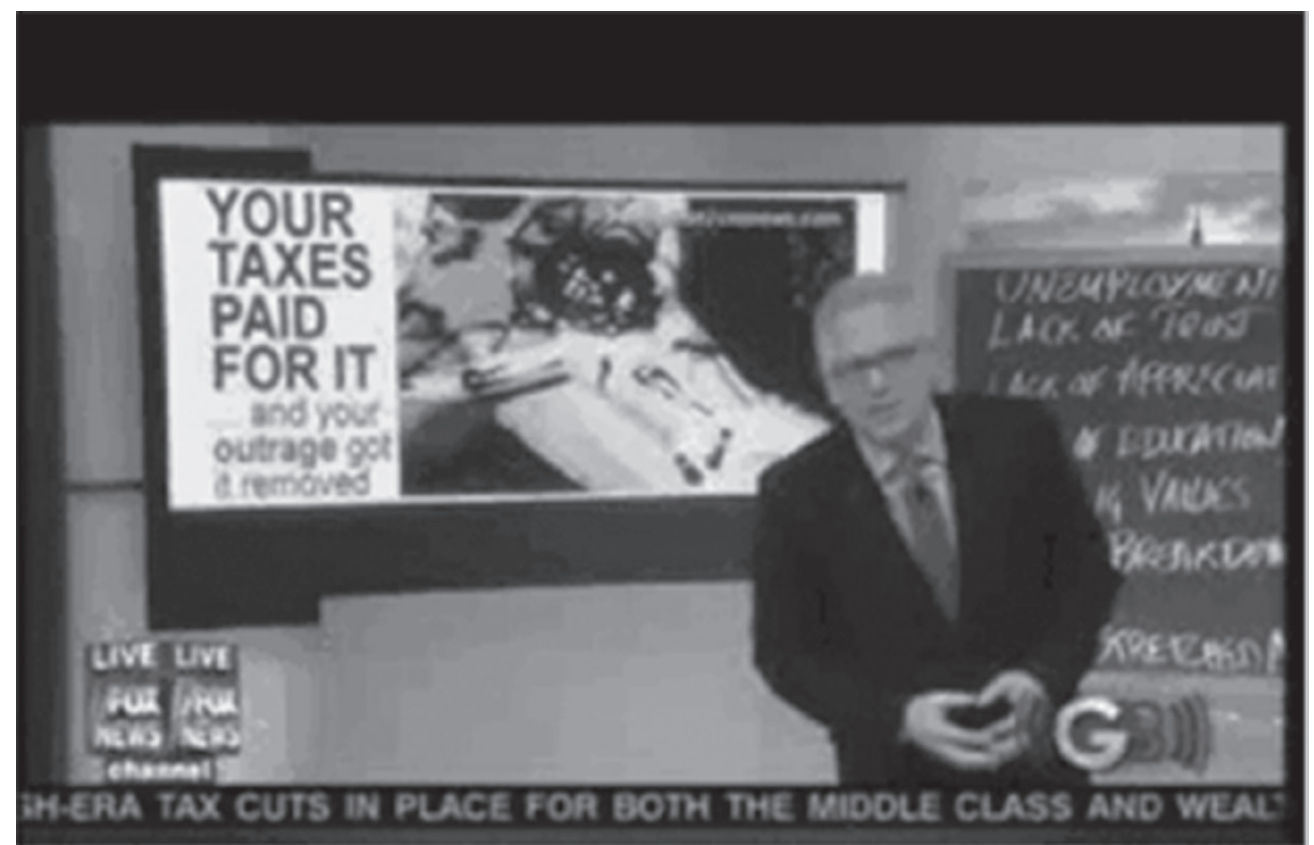

Illustration 2

Glenn Beck, Fox News, 30 novembre 2010.

<http://mediamatters.org/research/201011300052?utm_ source=twitterfeed\&utm_medium=twitter\&utm_campai gn $=$ Feed $\% 3 \mathrm{~A}+$ +mediamatters $\% 2$ Flatest $+\% 28$ Media+Matte rs+-+Latest+Items\%29> (consulté en juin 2011). 


\section{Méthodologie}

L'approche méthodologique adoptée pour cet article s'inspire de la sociologie de la médiation ou des associations, également connue sous le nom de théorie de l'acteur-réseau ${ }^{5}$. D'abord appliquée à la sociologie des sciences et des techniques, cette théorie de l'enquête obtint également une ample résonnance dans le milieu des sciences sociales et peut être aisément transposable à différentes disciplines telles la muséologie et l'histoire de l'art. L'acteur ne doit pas être conçu ici au sens d'intermédiaire, mais bien comme un médiateur qui peut modifier le cours des choses de manière inattendue. Le réseau, quant à lui, désigne la chaîne tracée par les traductions opérées entre les acteurs ${ }^{6}$.

La sociologie de la médiation consiste à suivre et à décrire de manière concise les réseaux de liens qui se tissent entre acteurs et agents non humains ${ }^{7}$ dans une conjoncture donnée, à travers les controverses et leur stabilisation. Cet outil méthodologique permet de comprendre comment interagissent les acteurs dans la formation et l'escalade de la controverse ainsi que dans son éventuel apaisement. Il s'agit d'observer un phénomène en train de se faire et de relever les médiations et les traductions successives qui agissent sur la situation. Il suffit de suivre les acteurs eux-mêmes: "l'enquête commence par une médiation, suit ou enfile les traductions et tombe sur une série de surprises où se noue et se dénoue la question même des acteurs et de leur réseau ${ }^{8}$."

Un des six principes fondamentaux de la théorie de l'acteur-réseau insiste sur l'importance de la traduction d'intérêts: «Les chercheurs et

5 Voir LATOUR, Bruno. Changer de société, refaire de la sociologie. Paris: La Découverte, 2006; et LATOUR, Bruno. La science en action. Paris: La Découverte, 1989. 6 Les acteurs forment un collectif, les uns faisant agir les autres; ainsi se tisse un réseau. (LATOUR, Changer de société, refaire de la sociologie, op. cit., p. 155).

7 La théorie de l'acteur-réseau intègre aussi les agents qui ne sont pas des personnes, c'est-à-dire tout objet ou toute chose qui agit au même titre que les humains à l'intérieur du phénomène étudié. (LATOUR, La science en action, op.cit., p. 202). les ingénieurs parlent au nom des nouveaux alliés qu'ils ont forgés et recrutés; porte-parole parmi les autres porte-parole, ils ajoutent ces ressources inattendues pour faire pencher la balance des forces en leur faveur ${ }^{9}$." Tout comme les experts de la science et du génie, parce qu'ils désirent tous atteindre leurs buts respectifs, les commissaires d'expositions, les artistes, les politiciens, les activistes ou les journalistes impliqués dans les controverses ajustent leurs intérêts mutuels et s'allient parfois, contre toute attente. Par exemple, un journaliste peut se rallier à la position d'un sénateur à propos d'une exposition controversée dans le but de hausser les ventes du périodique auquel il est affilié, le politicien employant lui-même cette polémique pour accroître sa visibilité en vue d'une élection prochaine. Pareillement, un musée, voulant conserver l'appui du gouvernement, peut décider de procéder au retrait d'une œuvre contestée, l'objet ayant lui-même été conçu pour déstabiliser un certain segment de la population. Il en résultera que l'artiste visé sera projeté sur la scène médiatique et, curieusement, que l'institution notera une nette hausse de sa fréquentation. C'est donc en créant des associations, seraient-elles transitoires, que les acteurs parviennent à faire tourner les événements en leur faveur, ce qui engendre un dénouement parfois inattendu.

L'argumentation s'appuie sur les divers médiateurs impliqués dans le débat et les diverses médiations - envisagés comme autant d'opérateurs de transformations ${ }^{10}$ - afin de déterminer comment, par une série de traductions d'intérêts, un discours est orienté et de quel ordre sont les interactions dans une situation polémique. En somme, cet article procède au suivi d'un réseau d'acteurs, réalisé à partir d'articles

8 LATOUR, Bruno. "Avoir ou ne pas avoir de réseau: that's the question". In. AKRICH, Madeleine, Yannick BARTHE, Fabian MUNIESA et Philippe MUSTAR (dir.). Débordements, mélanges offerts à Michel Callon. Paris: Presses de l'École des Mines, 2010, p. 258.

9 LATOUR, La science en action, op. cit., p. 428.

10 Selon la sociologue de l'art Nathalie HEINICH (La sociologie de l'art, Paris: La Découverte, 2001, p. 66), les médiateurs constituent des opérateurs de transformation ou de traduction, c'est-à-dire qu'ils ont la capacité de modifier l'issue d'une situation. 
de périodiques et d'observations sur les réseaux sociaux, permettant ainsi de " retracer $^{11}$ " la chaîne de médiations liant les intervenants entre eux.

\section{Entre art et activisme: l'œuvre de David Wojnarowicz}

David Wojnarowicz (1954-1992) fut actif sur la scène new-yorkaise entre la fin des années 1970 et le début des années 1990. Sa production artistique résolument engagée transcende les catégories de médiums traditionnelles, offrant une grande perméabilité entre la vidéo, la performance, la photographie, la peinture et le collage. Décédé des suites du SIDA en 1992, Wojnarowicz était également un activiste très impliqué dans la cause des droits des homosexuels et des individus atteints du VIH, alors marginalisés et fortement discriminés par les autorités politiques et religieuses. Ses propos irrévérencieux en ont d'ailleurs fait un acteur important des guerres culturelles au tournant des années 1990, alors qu'il luttait à la fois pour la liberté artistique et sexuelle: « My rage is really about the fact that when I was told that I'd contracted this virus it didn't take me long to realize that I'd contracted a diseased society as well ${ }^{12}$."

\section{A Fire in My Belly}

L'œuvre à l'origine de la controverse autour de Hide/Seek, A Fire in My Belly (1987), est un collage vidéographique rendant hommage à Peter Hujar, artiste, ami et mentor de Wojnarowicz décédé du SIDA en 1987. S'inspirant de

11 LATOUR, Changer de société. Refaire de la sociologie, op. cit., p. 187.

12 WOJNAROWICZ, David. «X Rays from Hell». In. Close to the Knives. A Memoir of Disintegration. New York: Vintage Books, 1991, p. 114. Wojnarowicz était un fervent défenseur de la lutte pour le financement public des arts et il prit part de manière active à la controverse entourant l'exposition The Perfect Moment du photographe Robert Mapplethorpe, qui avait déclenché un débat national sur le National Endowment for the Arts, la seule instance gouvernementale qui subventionne la création artistique aux États-Unis. Voir à ce sujet BOLTON, Richard (dir). Culture Wars: Documents from the Recent Controversies in the Arts. New York: The New Press, 1992. l'imagerie latino-américaine, l'œuvre constitue un montage de diverses scènes tournées au Mexique montrant, entre autres, une séquence de onze secondes où des fourmis se déplacent sur un Christ de plastique bon marché posé au sol (ill. 1). Établissant un parallèle entre la souffrance de l'humanité et celle de Jésus, cette image fait référence à l'épidémie de SIDA qui décimait littéralement l'entourage de l'artiste. Les insectes revêtent un rôle important dans la production de Wojnarowicz: «I used the ants as a metaphor for society because the social structure of the ant world is parallel to ours ${ }^{13}$." C'est donc un Christ en relation avec l'humain que l'artiste présente au regardeur dans A Fire in My Belly et non une vision volontairement blasphématoire.

\section{Exposition et réception critique}

Située à Washington (DC), la National Portrait Gallery est un musée fédéral assez conservateur, connu davantage pour ses tableaux de présidents et de héros de la culture populaire que pour son audace en matière de choix curatoriaux ${ }^{14}$. Hide/Seek y faisait exception.

Organisée par deux commissaires chevronnés, Jonathan D. Katz, éminent chercheur en études gaies et lesbiennes, et David C. Ward, historien, elle constituait la première exposition d'envergure à aborder l'apport des artistes homosexuels dans le genre du portrait moderne aux États-Unis. Rassemblant une centaine d'œuvres retraçant tout le $\mathrm{XX}^{\mathrm{e}}$ siècle, l'exposition avait pour objectif de jeter un regard nouveau sur une dimension négligée de l'histoire de l'art américain ${ }^{15}$.

13 PPOW GALLERY. Statement in Response to the Removal of David Wojnarowicz's "A Fire in my Belly" from Smithsonian National Portrait Gallery's Exhibition. <http://www.ppowgallery.com/press_release.php?id=87> (consulté en décembre 2010).

14 MEISLER, Stanley. "'Hide/Seek': National Portrait Gallery Exhibition of Homosexual Art ". Los Angeles Times, 14 novembre 2010. <http://articles.latimes.com/print/ 2010/nov/14/entertainment/la-ca-gay-portraits-20101114> (consulté en janvier 2011).

15 KATZ, Jonathan D. et David C. WARD. Hide/Seek: Difference and Desire in American Portraiture. Washington (DC): Smithsonian Books, 2010, p. 9. 


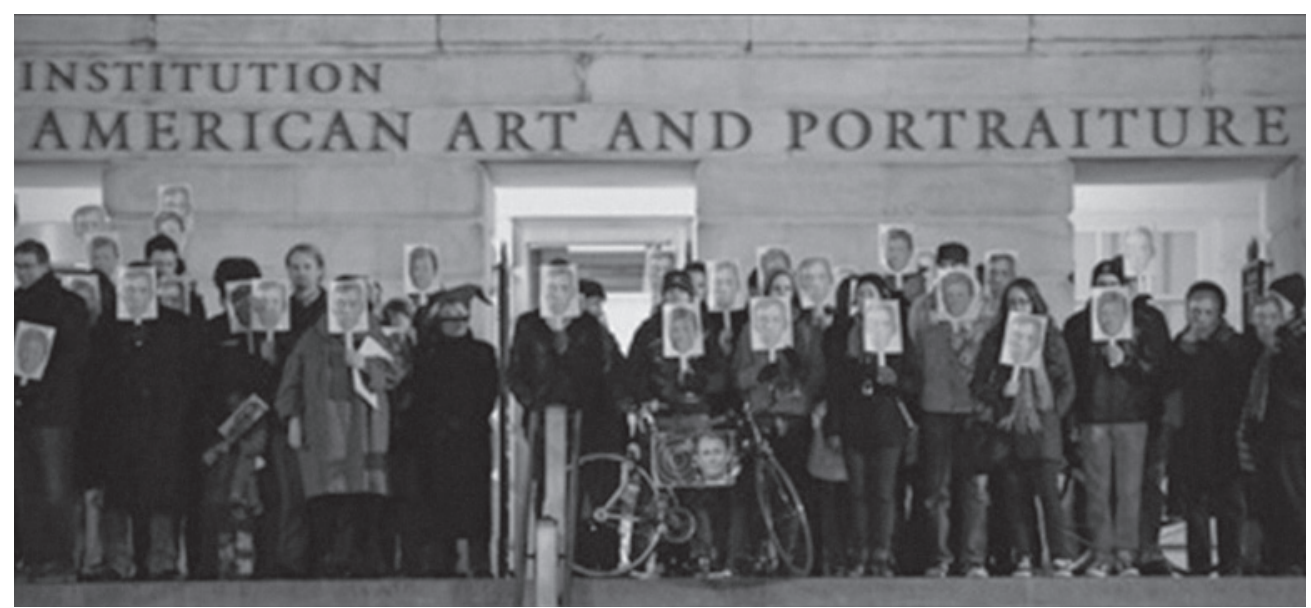

Illustration 3

Action silencieuse devant la National Portrait Gallery organisée par la galerie Transformer, 2 décembre 2010.

<https://www.facebook.com/photo.php?fbid=16519542 $6850103 \&$ set $=$ pu. $164716430231336 \&$ type $=1 \&$ theater $>$ (consulté en juillet 2011). 


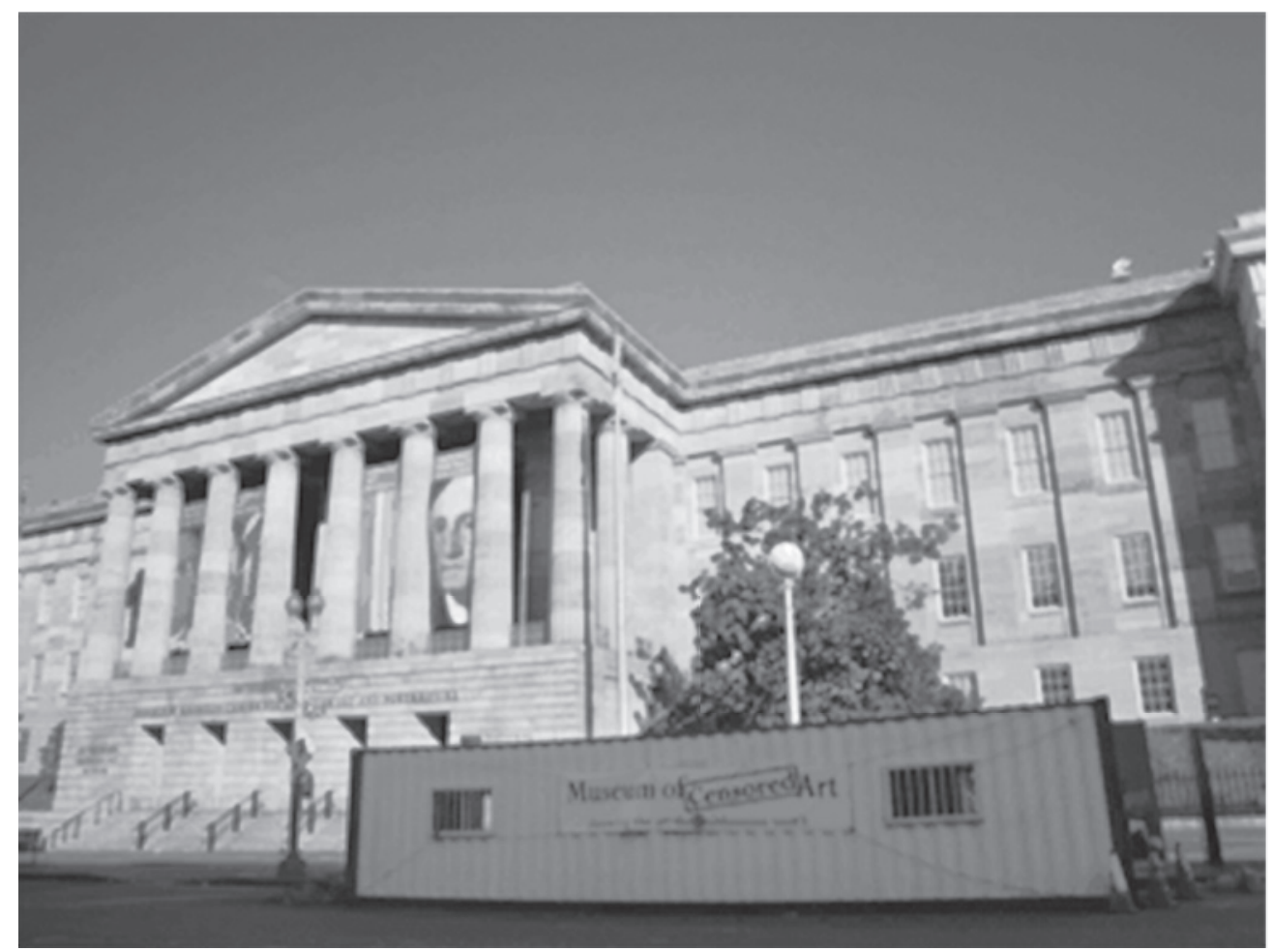

Illustration 4

Le Museum of Censored Art devant

la National Portrait Gallery, février 2011.

Photo: Julia Roberge Van Der Donckt 
La NPG est un des rares musées étasuniens à bénéficier de financement fédéral. Or, ces fonds servent au fonctionnement général du musée et non à la dizaine d'expositions temporaires qui y sont organisées chaque année. Les coûts de Hide/Seek - l'exposition la plus chère jamais présentée par l'institution avec un budget s'élevant à $750000 \$ \$^{16}$ - furent entièrement défrayés par le secteur privé, notamment la Mapplethorpe Foundation, la Calder Foundation et l'Andy Warhol Foundation for the Visual Arts.

Par ailleurs, Hide/Seek bénéficia d'une excellente réception critique. Bien que la sélection d'œuvres était assez prudente, un avertissement signalant aux visiteurs la nature adulte du contenu de l'exposition a tout de même été placé à l'entrée. Enfin, aucun incident ne fut signalé durant le premier mois de l'exposition.

\section{Déclenchement de la controverse}

La controverse autour de Hide/Seek s'est enflammée le 29 novembre 2010 lorsque le site Internet conservateur Cybercast News Service (CNS), connu précédemment sous le nom de Christian News Service, fit paraître un article critiquant de manière virulente l'exposition, texte signé par Penny Starr, rédactrice et activiste conservatrice ${ }^{17}$. Il faut signaler que CNS est le portail de nouvelles du Media Research Center (MRC), la plus grande organisation de surveillance et d'analyse de contenu médiatique aux États-Unis ${ }^{18}$.

En outre, il est intéressant de noter que Brent Bozell III, fondateur et président du MRC, siégeait également à ce moment au conseil

16 TRESCOTT, Jacqueline. «Portrait Gallery Removes Crucifix Video from Exhibit after Complaints ». The Washington Post, $1^{\text {er }}$ décembre 2010. <http://www. washingtonpost.com/wpdyn/content/article/2010/11/30/ AR2010113006801.html> (consulté en décembre 2010). 17 STARR, Penny. «Smithsonian Christmas-Season Exhibit Features Ant-covered Jesus, Naked Brothers Kissing, Genitalia, and Ellen DeGeneres Grabbing Her Breasts ». CNS News, 29 novembre 2010. <http://www.cnsnews.com/ news/article/smithsonian-christmas-season-exhibit-fea> (consulté en décembre 2010). d'administration de la Catholic League for Religious and Civil Rights, un groupe d'intérêt fondamentaliste très influent aux États-Unis. Bozell exprima son indignation à l'égard de l'exposition: "This federally-funded vulgarity by the Smithsonian Institution must come to an end immediately. How dare anyone use a federal facility—The Smithsonian's National Portrait Gallery no less- to exhibit such obscene material $^{19}$."

Afin de diffuser son article, qu'il convient de considérer comme un acteur non humain important dans le cadre de cette étude, et de provoquer un éventuel tollé chez les républicains, Starr envoya un courrier électronique à certains membres de la Chambre des représentants et du Sénat en leur demandant si l'exposition devait être annulée, considérant sa nature répréhensible. Tout comme Bozell, elle induisit ses destinataires en erreur, laissant entendre que l'exposition avait été financée par l'argent des contribuables.

Aussitôt alertés par le courriel de Starr, deux politiciens d'allégeance républicaine, John Boehner, alors représentant de l'Ohio au Congrès pressenti pour devenir le porte-parole de son parti à la Chambre des représentants ${ }^{20}$, et Eric Cantor, représentant de la Virginie, adressèrent publiquement des reproches à l'intention du Smithsonian. Boehner menaça implicitement de demander une réduction du financement public attribué à l'institution: "Smithsonian officials should either acknowledge the mistake and correct it, or be prepared to face tough scrutiny beginning in January when the new majority in the House moves to end the job-killing spending spree in Washington ${ }^{21}$. " Cantor, de son côté, pressa la NPG d'annuler l'exposition.

18 La mission du Media Research Center est d'éduquer le public et les médias à propos des biais libéraux dans les médias. (MEDIA RESEARCH CENTER. About. <http:// www.mrc.org/about/about.aspx> [consulté en juillet 2011].) 19 STARR, Penny. "Congressman: Close Tax-Funded Smithsonian Exhibit Featuring Naked Brothers Kissing, Men in Chains--Despite Removal of Ant-Covered Jesus ". CNS News, 2 décembre 2010. <http://www.cnsnews.com/ news/article/congressman-calls-closing-taxpayer-funde> (consulté en juillet 2011).

20 Poste qu'il obtint effectivement le 5 janvier 2011. 
Les deux politiciens mirent l'accent sur l'un des enjeux dominants chez les acteurs de droite dans cette controverse, soit le financement public des musées.

La Catholic League for Religious and Civil Rights a également été un acteur décisif dans le débat touchant Hide/Seek. Dans un communiqué de presse émis le 30 décembre, l'organisation s'opposa vivement à l'exposition et cibla précisément A Fire in My Belly comme élément subversif en qualifiant l'œuvre de discours haineux antichrétien. Après s'être attardée longuement à discuter de la nature immorale de la vidéo, la ligue signala qu'elle allait faire pression sur le gouvernement afin que le financement accordé au Smithsonian soit reconsidéré ${ }^{22}$. De plus, le président du groupe d'intérêt et guerrier culturel, William Donohue, fit publier sur Internet quelques jours plus tard l'adresse électronique personnelle de l'un des commissaires, Jonathan D. Katz. Celui-ci reçut subséquemment plusieurs messages hostiles de nature antisémite et homophobe ${ }^{23}$.

De son côté, la chaîne de télévision Fox News, reconnue pour son sensationnalisme et son contenu de type infodivertissement, s'empressa de parler de la controverse naissante le 30 novembre, le jour même où le débat parut dans les médias. Glenn Beck, commentateur politique à la personnalité flamboyante et aux visées ultraconservatrices, manipula les faits en stipulant que l'exposition avait été payée avec l'argent des contribuables. Employant en arrière-fond l'image désormais célèbre du Christ recouvert de fourmis de Wojnarowicz (ill. 2), Beck tenta d'établir un parallèle entre

21 STARR, Penny. «Boehner and Cantor to Smithsonian: Pull Exhibit Featuring Ant-Covered Jesus or Else ».

CNS News, 30 novembre 2010. <http://www.cnsnews.com/ news/article/boehner-and-cantor-smithsonian-pull-exhi> (consulté en juillet 2011).

22 CATHOLIC LEAGUE FOR RELIGIOUS AND CIVIL RIGHTS. Smithsonian Hosts Anti-Christian Exhibit. <http://www.catholicleague.org/release.php?id=2033> (consulté en juillet 2011).

23 JUDKIS, Maura. "Hide/Seek Curator Jonathan Katz on Gay's Art Newest Threat: The left ». TBD News, 22 décembre 2010. <http://www.tbd.com/blogs/tbd-arts/2010/12/hideseek-curator-jonathan-katz-on-gay-art-s-newest-threat-theleft-6342.html> (consulté le 12 janvier 2011). l'intérêt décroissant des Américains envers la religion et le prétendu élitisme des institutions culturelles. Enfin, en avançant que A Fire in My Belly avait sciemment été conçue pour insulter les chrétiens et que sa présentation lors de la période de Noël à Washington n'était pas anodine, Beck parvint sans doute à convaincre plusieurs de ses téléspectateurs du caractère immoral et blasphématoire de l'exposition de la National Portrait Gallery. L'œuvre de Wojnarowicz constituait ainsi une cible tout indiquée pour alimenter la suspicion envers les institutions muséales, dont le contenu est soi-disant biaisé, et par le fait même promouvoir les intérêts de Beck et de Fox News.

Les propos enflammés de ces acteurs mentionnés précédemment paraissaient parfois mal informés, et pour cause. Aucun des commentateurs n'avait alors visité l'exposition. Starr s'est sans doute basée uniquement sur les communiqués de presse de la NPG présentant l'exposition pour écrire son article et tous les autres acteurs se sont apparemment fiés à sa critique publiée par CNS News. Le rôle de Starr par l'intermédiaire de Cybercast News Service dans la création de la controverse fut toutefois passé sous silence par la plupart des articles couvrant l'affaire, qui mentionnaient seulement les points de vue des détracteurs de l'exposition comme Cantor et Boehner ${ }^{24}$. Or, il est clair que la journaliste a été l'instigatrice du débat autour de Hide/Seek et il est probable que la controverse n'aurait pas eu lieu si ce médiateur d'importance n'avait pas écrit le 29 novembre 2010 cet article qui suscita un tollé médiatique. C'est à partir de cette première traduction que les maillons de la chaîne se sont attachés.

24 CAPPS, Kriston. "A Fire in Her Belly: Penny Starr, the Conservative Activist Who Punked the Smithsonian ". Washington City Paper, 8 décembre 2010. <http:// www.washingtoncitypaper.com/blogs/artsdesk/visualarts/2010/12/08/a-fire-in-her-belly-penny-starr-theconservative-activist-who-punked-the-smithsonian/> (consulté en juillet 2011). 


\section{Une décision hâtive}

Les stratégies de CNS News et des autres acteurs impliqués dans la polémique se révélèrent particulièrement efficaces. Le 30 novembre 2010, soit à peine 24 heures après la publication de l'article de Penny Starr, Wayne Clough, secrétaire général de l'institution Smithsonian, céda aux pressions de la droite et fit retirer A Fire in My Belly de Hide/Seek. Les commissaires ainsi que le directeur de la National Portrait Gallery, Martin Sullivan, s'opposèrent à cette décision, mais leur avis ne fut apparemment pas pris en compte. Sullivan révéla au Washington City Paper ses regrets face à la situation et avoua que l'institution s'était pliée aux demandes de Boehner, de Cantor et de la Catholic League par crainte de représailles après l'élection d'un gouvernement républicain majoritaire en janvier 2011 : «This was a decision we were not happy about doing [...] None of us wanted to do this. It was really not about the piece. The Smithsonian has so many priorities and needs. With the new Congress coming in, we have pressures to work with the team ${ }^{25}$."

Il est également impératif de situer cette décision hâtive dans le contexte général de l'institution. Clough est arrivé à la tête du Smithsonian en 2008 à la suite de scandales concernant des irrégularités dans la gestion budgétaire. De plus, à l'automne 2010, l'institution déficitaire préparait la construction de deux nouveaux musées. Le moment était donc particulièrement mal venu pour une controverse impliquant un prétendu mauvais emploi de financement public, épisode qui pourrait nuire sensiblement aux projets de l'institution. Or, le débat ne put être esquivé, ce qui mit le Smithsonian dans une position très délicate. Au lieu d'étouffer l'affaire, la censure de l'œuvre ne fit qu'alimenter le débat en attirant davantage l'attention sur Hide/Seek, ce qui engendra des médiations parfois inattendues de la part d'acteurs de différents horizons poursuivant par leurs intérêts propres.

25 CAPPS, Kriston. "Smithsonian Secretary Pulled NPG Video, But Why?». Washington City Paper, $1^{\text {er }}$ décembre 2010. <http://www.washingtoncitypaper.com/blogs/artsdesk/ visual-arts/2010/12/01/smithsonian-secretary-pulled-npgvideo-but-why/> (consulté en avril 2011).
Par ailleurs, considérant la rapidité avec laquelle A Fire in My Belly fut retirée de l'exposition, il est étonnant de constater qu'il n'y ait eu aucune interaction directe entre les différents individus qui avaient demandé des changements à l'exposition et la direction du musée. Certes, les pressions politiques qui pesaient sur une institution publique comme le Smithsonian étaient non négligeables. Cependant, Clough céda très rapidement face à des critiques injustifiées alors qu'il aurait pu privilégier le dialogue et les échanges publics. La crédibilité de la National Portrait Gallery et du Smithsonian fut fortement ébranlée par cette affaire, faisant oublier la témérité de l'institution qui présenta une exposition dont le contenu potentiellement polémique dissuada plusieurs autres musées ${ }^{26}$.

\section{Stratégies de riposte du monde des arts et du milieu progressiste}

Le $1^{\text {er }}$ décembre 2010 , soit la journée internationale du SIDA, la vidéo de Wojnarowicz était disparue de l'exposition Hide/Seek. Pour plusieurs activistes, il s'agissait d'un geste lourd de sens, compte tenu de la nature de l'œuvre et du fait que son créateur était un ardent militant pour les droits des personnes atteintes du SIDA. Alors que les orthodoxes culturels ${ }^{27}$ voyaient en l'exposition de la NPG une tentative de gâcher le Noël des Américains aux valeurs traditionnelles, le milieu progressiste suspectait la décision de Clough d'être une stratégie pour faire taire les artistes homosexuels.

Le jour même du retrait de l'œuvre, People for the American Way, un groupe d'intérêt libéral défendant la liberté d'expression très actif sur la scène politique et culturelle, diffusa un communiqué de presse déplorant fortement la décision du Smithsonian: "That Bill [William] Donohue and the Catholic League are fighting for censorship is unsurprising. That the GOP [Grand

26 JUDKIS, "Hide/Seek Curator Jonathan Katz", op. cit. 27 HUNTER, Culture Wars: The Struggle..., op. cit., p. 45. 
Old Party] is echoing their call is shameful. That the Smithsonian has given into their transparent political bullying is deeply disturbing ${ }^{28}$."

La galerie Transformer de Washington (DC), un organisme à but non lucratif soutenant les artistes émergents à l'échelle locale et internationale, fut toutefois le premier acteur à réaliser une intervention concrète dans cette affaire. Initiant le dialogue autour du retrait de la vidéo de Wojnarowicz, la directrice Victoria Reis installa l'œuvre dans la vitrine de ses locaux le $1^{\text {er }}$ décembre afin que A Fire in My Belly puisse être vue par un maximum de personnes. Le lendemain, la galerie organisa une manifestation silencieuse devant la National Portrait Gallery, à laquelle une centaine de personnes participa (ill. 3).

Dans une vaste démonstration de solidarité, plusieurs galeries d'art et institutions muséales suivirent ensuite l'exemple de la Transformer en exposant l'œuvre censurée, dont le New Museum de New York et la Tate Gallery de Londres. Cette opération fut facilitée par la galerie PPOW, représentant les ayants droit de Wojnarowicz. Cette dernière annonça qu'elle autorisait la présentation publique de A Fire in My Belly à tous ceux qui le désiraient. En janvier, le Museum of Modern Art de New York (MoMA) créa un précédent en annonçant l'acquisition de l'œuvre de Wojnarowicz. Il s'agissait du premier musée à intégrer la vidéo à sa collection.

Les différents acteurs du milieu artistique s'étant concertés pour permettre la présentation de l'œuvre censurée de Wojnarowicz ont finalement réussi à faire en sorte que l'œuvre soit diffusée à une échelle bien plus large

28 PEOPLE FOR THE AMERICAN WAY. "Smithsonian Gives in to the Far Right, Censors the Artist Who "Spoiled Christmas" ", 2 décembre 2010. <http://67.192.238.60/pressreleases/2010/12/smithsonian-gives-to-the-far-right-censorsthe-artist-who-spoiled-christmas> (consulté en juillet 2011). 29 THE ANDY WARHOL FOUNDATION FOR THE VISUAL ARTS. «Warhol Foundation Demands Reinstatement of Censored Art Work or Will Cease Funding all Smithsonian Institution Exhibitions ", 13 décembre 2010. $<$ http://warholfoundation.org/foundation/35_detail. html? page $=1>($ consulté en juillet 2011) . que ce qui avait été prévu initialement par la National Portrait Gallery, contribuant ainsi à la renommée de l'artiste défunt. En outre, les galeries et les institutions impliquées ont certainement profité de cette occasion pour accroître leur fréquentation, la controverse étant un outil publicitaire avéré. L'engagement de la galerie Transformer dans la controverse entourant Hide/Seek lui permit donc d'asseoir sa réputation d'acteur de premier ordre dans le soutien des arts visuels dans la capitale américaine.

La stratégie de réplique du monde des arts prit aussi la forme de mesures visant à pénaliser la NPG. Condamnée par l'Association of Art Museum Directors, la censure de A Fire in My Belly fut également décriée par certains mécènes du musée. À la mi-décembre, l’Andy Warhol Foundation for the Visual Arts, qui avait offert 375000 \$ à la National Portrait Gallery pour diverses expositions au cours des dernières années, dont Hide/Seek, réclama le retour de la vidéo dans l'exposition, sans quoi elle cesserait tout financement des expositions du groupe Smithsonian ${ }^{29}$. La Fondation Mapplethorpe et la fondation Calder ont également fait pression sur l'institution, l'une réduisant son soutien financier au musée et l'autre se désistant concernant le prêt d'une œuvre pour une exposition ultérieure ${ }^{30}$.

\section{Par ailleurs, l'artiste canadien AA Bronson} demanda sans succès que l'une de ses œuvres soit retirée de l'exposition en guise de solidarité avec Wojnarowicz. Un prêteur, le collectionneur Jim Hedges, tenta une démarche similaire à celle de Bronson, mais la NPG ne donna pas suite à sa requête ${ }^{31}$.

30 CAPPS, Kriston. "Mapplethorpe Foundation Withdraws Support for Smithsonian Exhibitions ". Washington City Paper, 17 décembre 2010. <http:// www.washingtoncitypaper.com/blogs/artsdesk/visualarts/2010/12/17/mapplethorpe-foundation-withdrawssupport-for-smithsonian-exhibitions/> (consulté en juillet 2011).

31 HEDGES, Jim. "Smithsonian Stands Firm on Censorship, Congressional Checkbook Trumps Constitution ". The Huffington Post, 11 janvier 2011. <http:// www.huffingtonpost.com/jim-hedges/smithsonian-standsfirm-o_b_807094.html> (consulté en janvier 2011). 
Les dissensions suscitées par Hide/Seek se firent sentir au sein même du Smithsonian. En effet, non seulement plusieurs employés signalèrent publiquement leur désaccord avec la décision de Clough, mais James T. Bartlett démissionna du comité consultatif de l'institution en geste de protestation ${ }^{32}$.

\section{De nouveaux modes de diffusion}

Il est indéniable qu'Internet a joué un rôle prééminent dans la mise en place du débat concernant la National Portrait Gallery. Permettant des interactions de type nouveau entre les acteurs, une vitesse de diffusion accrue et un public récepteur beaucoup plus large que ce que permettaient à eux seuls les médias traditionnels, Internet a modifié substantiellement le développement des controverses, densifiant les réseaux de médiation dans un contexte de guerres culturelles.

Tout d'abord, il faut rappeler que l'article de Starr qui amorça la controverse fut diffusé par l'intermédiaire d'un périodique en ligne et que les politiciens qui réclamèrent des modifications à Hide/Seek furent informés du contenu de l'exposition par courrier électronique. Les réseaux sociaux furent également déterminants dans la suite des événements. Ainsi, le jour même du retrait de A Fire in My Belly, une page Facebook intitulée "Support Hide/Seek " fut créée dans le but d'offrir un lieu de ralliement virtuel à ceux qui s'opposaient à la censure de l'exposition. L'identité des auteurs n'a pas été révélée, mais il s'agissait sans doute d'activistes du milieu artistique. À la manière d'un fil de nouvelles, la page diffusa pendant plusieurs mois les nouveaux développements de l'affaire et elle relaya les diverses interventions militantes menées après le retrait de l'œuvre, dont les projections de la vidéo de Wojnarowicz qui eurent lieu un peu partout aux États-Unis et ailleurs. Des milliers

32 TRESCOTT, Jacqueline. «Board Member Resigns Over Crucifix Controversy ». The Washington Post, 9 décembre 2010. <http://voices.washingtonpost.com/arts-post/2010/12/ portrait_gallery_director_issu.html > (consulté en juillet 2011). de personnes avaient ainsi accès à cette plateforme unique concentrant l'information la plus récente sur l'exposition et pouvaient laisser des commentaires. Le réseau Twitter fut également investi par plusieurs acteurs impliqués dans la controverse sur l'exposition Hide/Seek, tel Tyler Green, rédacteur pour le magazine en ligne ARTINFO qui avait fait une couverture extensive des événements entourant le retrait de l'œuvre de Wojnarowicz de la NPG.

Ces nouveaux modes de communication contribuèrent sans doute à amplifier l'implication de la population américaine dans le mouvement de réponse à la censure du Smithsonian. En somme, bien qu'il soit pour l'instant impossible de quantifier de manière exacte l'incidence d'Internet et des réseaux sociaux dans le développement de la controverse, il est certain que ce média permit d'étendre de manière substantielle les châ̂nes d'interactions formées par les différents acteurs impliqués.

Un autre type de technologie rendit possible la réalisation d'une intervention inusitée à la National Portrait Gallery qui eut de fortes répercussions sur la suite du débat. Le 4 décembre 2010, Michael Blasenstein, webmestre et militant pour les droits des homosexuels, se présenta près de l'entrée de la salle d'exposition de Hide/Seek muni d'une tablette électronique $i P a d$ diffusant un extrait de A Fire in My Belly et présentant des dépliants expliquant aux visiteurs les motifs de son entreprise. Pendant ce temps, son acolyte, l'artiste photographe Michael Iacovone, filmait l'opération visant à réintégrer l'œuvre censurée de Wojnarowicz dans l'exposition.

L'action fut rapidement interrompue par les agents de sécurité du musée, qui interpellèrent Blasentein, stipulant qu'il était interdit de distribuer des brochures dans l'institution. En guise de réprimande, les deux activistes furent 
bannis de toutes les institutions Smithsonian pour une période d'un $\mathrm{an}^{33}$. En dépit de l'interdiction de fréquenter la National Portrait Gallery, Blasenstein et Iacovone trouvèrent une stratégie encore plus efficace pour faire valoir leur désaccord face au retrait inopiné de A Fire in My Belly.

\section{Le Museum of Censored Art}

Cette fois armé de permis, le duo d'activistes élabora un projet d'exposition qui faisait un pied de nez au Smithsonian. L'irrévérencieux Museum of Censored Art ouvrit ses portes le 13 janvier 2011, soit exactement un mois avant la fin de Hide/Seek, tout près de l'une des entrées de la NPG. Employant un conteneur industriel en guise de local, Blasenstein et Iacovone y installèrent l'œuvre de Wojnarowicz ainsi que quelques panneaux informatifs. Ils incitaient ce faisant les passants à visiter leur création protestataire. Le mandat du musée improvisé, apposé sur une bannière bien en vue, était simple, mais téméraire: «showing the art that the Smitsonian won't " (ill. 4). Selon les créateurs, les buts de l'entreprise étaient de faire en sorte que le Smithsonian réintègre la vidéo de Wojnarowicz, de donner accès à l'œuvre censurée au public et de tenir l'institution responsable de ses actions. Afin d'offrir une alternative entièrement viable à la présentation de A Fire in My Belly à la NPG, le Museum of Censored Art observa les mêmes heures d'ouverture que son voisin et son accès était lui aussi gratuit.

Même si l'œuvre ne regagna pas les salles d'exposition du Smithsonian, Blasenstein et Iacovone se dirent néanmoins très satisfaits de leur réalisation. Publicisée notamment par l'entremise de Facebook et de Twitter, l'exposition a attiré 6476 visiteurs au cours des

33 GOPNIK, Blake. «Protestors Banned from Smtihsonian after Playing Video on iPad". The Washington Post, 6 décembre 2010. <http://voices.washingtonpost.com/arts-post/2010/12/ on_sunday_at_around_1.html> (consulté en janvier 2011). 34 MUSEUM OF CENSORED ART. Home. <http:/ dontcensor.us/> (consulté en juillet 2011). trente jours de son ouverture et les activistes ont bénéficié d'une couverture médiatique à l'échelle nationale ${ }^{34}$. Les nombreux opposants à la censure de la NPG trouvèrent ainsi une voix concrète en ce collectif temporaire.

\section{Le Museum of Censored Art constitua sans} contredit un épisode majeur de la controverse entourant Hide/Seek. Réunissant des acteurs déterminants, tels des militants pour la liberté artistique et l'œuvre de Wojnarowicz ellemême, cette intervention inaugura une stratégie de riposte inusitée alliant des techniques contestataires traditionnelles aux nouvelles possibilités des médias sociaux. À la manière d'un Salon des refusés du XXI ${ }^{\mathrm{e}}$ siècle, le Museum of Censored Art agit comme un agent subversif hautement efficace, défiant l'autorité du Smithsonian tout juste devant ses locaux.

\section{Stabilisation de la controverse}

Il n'est pas étonnant de constater que le débat hautement médiatisé sur Hide/Seek: Difference and Desire in American Portraiture fit en sorte que l'exposition eut un vif succès. En janvier 2011 uniquement, la NPG reçut plus de 85000 visiteurs $^{35}$. Toutefois, l'image de l'institution fut certainement ébranlée par la controverse. Clough fut d'ailleurs visé directement par le milieu de l'art, non seulement parce qu'il fléchit face aux pressions de la droite religieuse, mais également en raison de son manque de transparence. Le secrétaire général tarda en effet à commenter publiquement les événements de la fin novembre, accordant une première entrevue seulement deux mois après le retrait de l'œuvre de Wojnarowicz. Clough concéda que la décision avait été prise de manière précipitée, mais il déclara qu'il s'agissait de la meilleure chose à faire compte tenu de la conjoncture économique contraignante:

35 Il est cependant impossible de déterminer le nombre exact de visiteurs pour Hide/Seek, car l'accès au musée et à ses expositions temporaires est gratuit. (TRESCOTT, Jacqueline. "Hide/Seek Visitors Register Their Opinions". The Washington Post, 10 février 2011.<http://voices. washingtonpost.com/artspost/2011/02/hideseek_visitors_ register_the.html> [consulté en février 2011].) 
"We are going into a period which I would describe as probably the most difficult period for funding for federal agencies in our lifetime ${ }^{36}$."

Le 31 janvier 2011, le conseil des régents du Smithsonian annonça une série de nouvelles procédures concernant la présentation d'expositions susceptibles d'attirer la controverse. Il fut notamment recommandé de ne pas altérer le contenu d'une exposition à moins d'un accord entre les commissaires, le directeur du musée et l'administration de l'institution ${ }^{37}$.

La polémique commença à se stabiliser au moment où Hide/Seek se termina, soit à la mifévrier 2011. Or, il est impératif de souligner que l'implication du milieu conservateur fut extrêmement différente de celle du camp progressiste dans la chaîne d'interactions. Les interventions des orthodoxes culturels furent nombreuses, mais condensées au début du débat, soit de la fin novembre à la fin décembre 2010, tandis que celles des artistes et de diverses institutions culturelles s'étalèrent sur une période plus longue. Alors que le milieu de l'art continuait de s'enflammer, les commentaires des acteurs de la droite religieuse se firent assez rares après le retrait de A Fire in My Belly.

Il est possible d'en déduire qu'une fois que les conservateurs eurent atteint leur objectif, c'est-à-dire une réponse rapide du Smithsonian qui modifia immédiatement le contenu de Hide/Seek, ils ne crurent pas nécessaire de s'acharner sur l'institution. Certes, des menaces d'enquête budgétaire planèrent sur la NPG pendant quelque temps, mais celles-ci ne se concrétisèrent pas. En somme, les diverses stratégies de riposte élaborées par les progressistes parvinrent à maintenir la

36 TRESCOTT, Jacqueline. "Smithsonian Secretary Clough Stands by Decision to Pull 'Fire in My Belly' Video ". The Washington Post, 19 janvier 2011. <http://www. washingtonpost.com/wpdyn/content/article/2011/01/18/ AR2011011805097.html> (consulté en janvier 2011). 37 CULTURE MONSTER. «Smithsonian Announces New Procedures To Deal with Controversial Exhibitions ". Los Angeles Times, 31 janvier 2011. <http://latimesblogs.latimes. com/culturemonster/2011/01/smithsonian-announces-newprocedures-to-deal-with-controversial-exhibitions.html> (consulté en février 2011). controverse active sur une assez longue période.

Ces diverses entreprises s'avérèrent toutefois vaines, car Starr, Boehner, Cartor, Beck et la

Catholic League savouraient déjà leur victoire.

Chacun des acteurs impliqués dans le débat sur l'exposition Hide/Seek ont vu en cette controverse un moyen de promotion, voire de propagande. Les groupes de pression, politiciens et autres individus de toutes allégeances ont ainsi employé la polémique afin de promouvoir leurs intérêts respectifs, qu'ils fussent louables ou non. C'est en accumulant les tactiques de diffusion, des opérateurs de transformations comme la publication d'articles ou la réalisation d'actions protestataires, que les acteurs créèrent des associations et densifièrent par le fait même le réseau de médiations.

Malgré le débat virulent dont elle fut à l'origine (ou peut-être grâce à celui-ci), Hide/ Seek fut nommée la meilleure exposition thématique de l'année par l'Association of Art Museum Curators en mai 2011. Le Museum of Censored Art ne fut pas en reste, ayant été salué pour son audace. En mai 2011, l'American Library Association décerna à Michael Iacovone et Michael Blasenstein le prix John-Phillip-Immroth récompensant des projets défendant la liberté intellectuelle ${ }^{38}$.

\section{Conséquences de la polémique}

Contrairement à d'autres sphères où ils opèrent de manière continuelle, les conflits idéologiques affectant les États-Unis demeurent latents en ce qui concerne le domaine muséal. Se déclarant de façon épisodique ${ }^{39}$, ils rappellent aux institutions la portée du lobby

38 WILLIAMS, Erin. "Creators of Museum of Censored Art Receive Intellectual Freedom Award ». The Washington Post, 25 mai 2011.<http://www.washingtonpost.com/ lifestyle/style/creators-of-museum-of-censored-art-receiveintellectual-freedom-award/2011/05/25/AGgaITBH_story. html> (consulté en août 2011).

39 HEINICH, Nathalie. Guerre culturelle et art contemporain. Une comparaison franco-américaine. Paris: Hermann, 2010, p. 8. 
conservateur, mais aussi la solidarité du monde de l'art, les diverses factions employant une vaste gamme de stratégies pour arriver à leurs fins.

Lieu d'une convergence d'intérêts, l'exposition Hide/Seek: Difference and Desire in American Portraiture interpella un vaste réseau d'acteurs, créant ainsi une controverse qui tint en haleine les médias pendant plusieurs mois. Ce débat confirme de manière incontestable la récurrence des guerres culturelles sur la scène muséale étasunienne. Il est toutefois encore trop tôt pour en déterminer toutes les conséquences.

Or, il certain que cet épisode pourrait affecter le financement de futures expositions dont le contenu est considéré comme risqué, explorant des thèmes comme la religion, le patriotisme ou l'homosexualité, soit des terrains toujours sensibles aux États-Unis. Si l'on se fie aux propos de Boehner, Cantor, Donohue et Beck, la droite religieuse semble déterminée à poursuivre sa lutte contre le financement public des arts et des institutions culturelles entamée à la fin des années $1980^{40}$. Afin de mener à bien son projet, elle entend même désormais cibler des expositions dont les coûts sont entièrement défrayés par le secteur privé, dans le but de soulever l'ire des autorités et des contribuables envers des œuvres soi-disant blasphématoires. Cela entraîne inévitablement un climat d'autocensure chez les institutions muséales, soucieuses de préserver un financement qui est vital au fonctionnement de leur établissement.

L'exposition organisée par la National Portrait Gallery ne devait initialement pas voyager. Cependant, au printemps 2011, deux musées décidèrent de l'ajouter à leur programmation

40 Voir SMITH, David A. Money for Art. The Tangled Web of Art and Politics in American Democracy. Chicago: Ivan R. Dee, 2008.

41 En 1999, alors dans une situation financière difficile, le Brooklyn Museum avait présenté Sensation: Young British Artists from the Saatchi Collection, une exposition qui avait fait scandale lorsqu'elle avait été présentée à la Royal Academy of Art de Londres deux ans plus tôt. Une vaste controverse s'était alors déclenchée à New York et le musée avait affiché une fréquentation record. (Voir ROTHFIELD, Unsettling "Sensation.” Arts-Policy Lessons..., op. cit.) pour la saison 2011-2012. Hide/Seek sera ainsi présentée au Brooklyn Museum de novembre à février, alors que le Tacoma Art Museum situé dans l'État de Washington accueillera l'exposition de mars à juin. Il faut rappeler qu'il y a quelques années, lorsque les commissaires Katz et Ward préparaient Hide/Seek pour la NPG, aucun autre musée ne s'était montré intéressé par le projet, sans doute en raison de sa nature potentiellement polémique. La controverse de 2010-2011 changea la donne, l'exposition apparaissant comme une opération très rentable, assurée d'attirer un grand nombre de visiteurs et peut-être quelques fondamentalistes religieux et politiciens en mal de publicité au passage. Une réactivation du conflit est donc à envisager.

Considérant la relation ambivalente que les musées entretiennent avec la controverse, la fuyant par crainte de représailles ou l'alimentant dans une volonté de créer de la publicité, il sera intéressant de voir quelle position adopteront la direction du Brooklyn Museum et celle du Tacoma Art Museum ${ }^{41}$. 
Julia Roberge Van Der Donckt, translated by Allana Carlyle

Culture Wars and Controversy in American Art Museums: The case of Hide/Seek: Difference and Desire in American Portraiture

Starting at the end of the 1980's, American culture wars operated on multiple terrains and the museal sphere was no exception. Certain American institutions became the theatre for ideological struggles opposing progressives and conservatives. After about 10 years of relative tranquility, the museal world was again shaken by a large scale controversy in the winter of 2011, reactivating the debate on the public financing of the arts.

The controversy surrounding Hide/Seek: Difference and Desire in American Portraiture, presented at the National Portrait Gallery (NPG) in Washington D.C. from October $30^{\text {th }}, 2010$ to February $13^{\text {th }}, 2011$, significantly testifies to the incidents of cultural wars on art museums. As the first exhibition to tackle the contribution of homosexual artists in the genre of modern portraits in the United States, Hide/Seek took on sensitive subjects such as religion and the demands of the gay community.

Following bitter critique put out by influential conservative-right players, the video work A Fire in My Belly by David Wojnarowicz was hastily pulled from the exhibition by the General Secretary of the Smithsonian group, of which NPG is a member. The target of this impulse of hostility was a short sequence showing ants crawling on a crucified Jesus. It took no more than that to raise the ire of the catholic league and of some republican politicians lacking publicity who signalled their aversion to this alleged blasphemy.

From this, a vast public debate arose. Many artistic organizations, journalists, and activists contested what they considered to be an unjustified act of censorship, while detractors of the exhibition called for increased firmness regarding the financial management of the Smithsonian. A variety of strategies were then employed by the enemy factions ranging from menacing letters to the creation of the Museum of Censored Act, an anti-establishment action presenting the work of Wojnarowicz in an industrial container at the doors of the NPG. The internet and social media served as crucial tools for the conflict. Liberals and conservatives also used these tools extensively in order to facilitate the ample diffusion of their interventions and their convictions on the subject of the exhibition.

In order to better understand this controversy, it is necessary to determine the nature of the sociological mechanisms which put it in place as well as the exact role of the different players who intervened.

This article focuses on the sociology of the mediations with the goal of revealing the concise manner in which the dynamics operated between the individuals and collectives who took part in the debate. This theory 
of inquiry, equally known as the Actor-Network Theory, allows for the treatment of the data, that is the sources of current affairs, by "re-tracing" (Latour, 2006: 187) the chain of mediations linking the participants together.

The text therefore highlights the players implicated in the controversy through rigorous description of the relationships they maintain in order to show how they act and modify the parameters of the situation. In this way, the text will be presented in function of the mediations revealed, reassembling in a clear manner the path laid out by the mediators.

In summary, this case study will shine light on the creation of a recent controversy which based on profound evidence, will have consequences on the museal landscape of the United States. 